\title{
LES PEUPLES AUTOCHTONES AUX NATIONS UNIES : UN NOUVEL ACTEUR DANS LA FABRIQUE DES NORMES INTERNATIONALES
}

\author{
Irène Bellier
}

Presses de Sciences Po | Critique internationale

\author{
$2012 / 1-n^{\circ} 54$ \\ pages 61 à 80
}

ISSN 1290-7839

Article disponible en ligne à l'adresse:

http://www.cairn.info/revue-critique-internationale-2012-1-page-61.htm

Pour citer cet article :

Bellier Irène, « Les peuples autochtones aux Nations unies : un nouvel acteur dans la fabrique des normes internationales »,

Critique internationale, $2012 / 1 \mathrm{n}^{\circ} 54$, p. 61-80. DOI : 10.3917/crii.054.0061

Distribution électronique Cairn.info pour Presses de Sciences Po.

(C) Presses de Sciences Po. Tous droits réservés pour tous pays.

La reproduction ou représentation de cet article, notamment par photocopie, n'est autorisée que dans les limites des conditions générales d'utilisation du site ou, le cas échéant, des conditions générales de la licence souscrite par votre établissement. Toute autre reproduction ou représentation, en tout ou partie, sous quelque forme et de quelque manière que ce soit, est interdite sauf accord préalable et écrit de l'éditeur, en dehors des cas prévus par la législation en vigueur en France. II est précisé que son stockage dans une base de données est également interdit. 


\title{
Les peuples autochtones aux Nations unies: un nouvel acteur dans la fabrique des normes internationales
}

\author{
par Irène Bellier
}

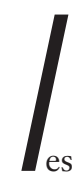

es représentants autochtones sont très actifs au sein des institutions internationales : à Genève et à New York, ils ont négocié la Déclaration des droits des peuples autochtones (DDPA, ci-après Déclaration); à Washington, ils parlementent avec la Banque mondiale ; à Paris, Genève et Rome, ils interviennent auprès de l'UNESCO, de l'OIT, de la Food and Agricultural Organization (FAO), du Fonds international de développement agricole (FIDA) ; à Rio, Durban, Tunis et Copenhague, ils participent aux conférences sur la biodiversité, le racisme, le changement climatique et la société de l'information. Dans toutes ces institutions et manifestations, ils portent la voix des peuples autochtones ${ }^{1}$ et agissent en qualité d'experts. Leurs revendications et les solutions qu'ils proposent ont mûri dans des réseaux transnationaux, des centres de recherches et dans l'activisme international. Celui-ci a pris le relais des mouvements sociaux observés dans

1. Nous mettons en italiques l'expression peuples autochtones pour signaler son usage comme catégorie, communément pluralisée. 
les Amériques à partir des années 1960, dans le Nord de l'Europe dans les années 1970, et plus récemment en Afrique, en Asie et dans les mondes anciennement communistes ${ }^{2}$. L'écho de ces luttes et la transformation du traitement institutionnel de « la question indigène $»^{3}$ nourrissent le mouvement international des peuples autochtones. Celui-ci est configuré de manière souple par les organisations autochtones qui se rattachent aux problématiques dont se saisissent les Nations unies.

L'adoption de la Déclaration, en 2007, par l'Assemblée générale (143/194 États) et le ralliement en 2009 et 2010 des quatre seuls opposants déclarés (Canada, Australie, Nouvelle-Zélande, États-Unis) marquent une étape significative de la manière dont les États prennent en considération les « sociétés indigènes /autochtones ». En posant leur droit à l'autodétermination (art. 3), la Déclaration scelle le principe de la reconnaissance des peuples autocbtones en droit international et ouvre la voie à la redéfinition de leur place dans la communauté internationale ${ }^{4}$. Les juristes ${ }^{5}$ se penchent sur «l'ambiguité constructive» de ce document, examinent la possibilité de mettre en œuvre ses dispositions et analysent la portée des droits collectifs énoncés, dont la plupart figurent dans le droit international ${ }^{6}$. Nous nous intéressons à l'influence du processus de la Déclaration sur la formation d'un imaginaire commun et à la manière dont ceux qui l'ont négociée poursuivent le travail sur les scènes régionales, nationales et locales.

Les organes des Nations unies qui inscrivent les sujets - individus et collectifs - ainsi que les problématiques autochtones à l'agenda de la planète

\footnotetext{
2. Voir, parmi de nombreuses publications, Alison Brysk, From Tribal Village to Global Village: Indian Rigbts and International Relations in Latin America, Stanford, Stanford University Press, 2000 ; Marisol de la Cadena, Orin Starn (eds), Indigenous Experience Today, New York, Berg, 2007 ; Yvon Le Bot, La grande révolte indienne, Paris, Robert Laffont, 2009 ; Françoise Morin, « Les Nations unies à l'épreuve des peuples autochtones », dans Christian Gros, Marie-Claude Strigler, Etre indien dans les Amériques - Spoliations et résistance. Mobilisations etbniques et politiques du multiculturalisme, Paris, Éditions de l'Institut des Amériques, 2006, p. 43-54 ; Tania Murray Li, The Will to Improve. Governmentality, Development, and the Practice of Politics, Durham, Duke University Press, 2007 ; Deborah Yashar, Contesting Citizenship in Latin America: The Rise of Indigenous Movements and the Postliberal Challenge, Cambridge, Cambridge University Press, 2005.

3. Isabelle Schulte-Tenckhoff, La question des peuples autochtones, Bruxelles, Bruylant, 1997 ; Jean-Claude Fritz, Frédéric Deroche, Raphaël Porteilla, La nouvelle question indigène. Peuples autochtones et ordre mondial, Paris, L'Harmattan, 2006.

4. S. James Anaya, Indigenous Peoples in International Law, New York, Oxford University Press, 2000.

5. Stephen Allen, Alexandra Xanthaki (eds), Reflections on the UN Declaration on the Rights of Indigenous Peoples, Oxford, Hart Publishing, 2011 ; Claire Charters, Rodolfo Stavenhagen (eds), Making the Declaration Work: The United Nations Declaration on the Rights of Indigenous Peoples, Copenhague, IWGIA (International Work Group for Indigenous Affairs), 2010.

6. Les deux pactes internationaux sur les droits civils et politiques et sur les droits culturels, économiques et sociaux, dont l'article 1 (commun) a inspiré l'article 3 de la Déclaration; la Convention 169 de l'OIT ; les Conventions sur l'élimination de toutes les formes de discrimination raciale (1969), sur l'élimination des discriminations à l'égard des femmes (1979), sur les droits de l'enfant (1989) et sur la diversité biologique (1992).
} 
constituent une plateforme où l'on observe les actions d'organisations variées, des processus de co-construction des savoirs et la production de normes. Celles-ci portent sur la reconnaissance de droits sur le territoire, l'usage des ressources naturelles, l'éducation, la justice ou la participation politique, et sont susceptibles de s'étendre. Le principe de l'association des acteurs autochtones à la fabrique de politiques et de normes qui peuvent affecter les sociétés locales nous semble significatif des transformations en cours. À partir d'une enquête fondée sur l'observation participante de plusieurs groupes de travail à Genève et à New York ${ }^{7}$, nous présenterons les modalités d'émergence du collectif peuples autochtones et relierons les formes de sa participation au débat relatif à la reconnaissance comme « peuple», sans attribut d'aucune sorte.

L'observation des scènes de dialogue et des sites d'interactions sur lesquels est pratiquée une ethnographie « dense ${ }^{8}$ nous conduit à analyser le rôle des leaders autochtones en interrogeant le régime discursif, les pratiques et les politiques qui marquent le changement de statut - de «victimes » sur les scènes locales à « acteurs » sur la scène internationale - et la formation d'une subjectivité partagée.

L'adoption des sociétés indigènes/autochtones par la Communauté internationale s'est traduite par la proclamation de l'Année internationale des populations autochtones en 1993 ; par celle, en 1994, du 9 août comme Journée internationale des populations autochtones; puis par celle, en 1995, de la première décennie internationale sur le thème « Populations autochtones : un partenariat dans l'action », suivie en 2005 d'une seconde décennie sur le thème du «Partenariat pour l'action et la dignité ». Apparemment symboliques, ces proclamations traduisent cependant un nouvel esprit. La familiarité des délégués autochtones non seulement entre eux mais aussi avec

\footnotetext{
7. L'auteur suit depuis 2002 les rendez-vous annuels de quatre organes des Nations unies spécialisés dans les questions autochtones, et ce pour des sessions de travail allant de une à deux semaines : à Genève, de 2002 à 2006, le Groupe de travail sur les populations autochtones (GTPA), créé en 1982, et le Groupe de travail sur le projet de Déclaration (GTPD), créé en 1995, tous deux dissous après l'adoption de la Déclaration par le Conseil des Droits de l'Homme; à New York, depuis 2002, l'Instance permanente sur les questions autochtones ou UNPFII (United Nations Permanent Forum on Indigenous Issues), sigle agréé en français ; à Genève, depuis 2008, le Mécanisme expert sur les droits des peuples autochtones (MEDPA). Ce suivi implique de participer aux sessions plénières et aux « événements parallèles », informels pour les Nations unies et essentiels à la construction du collectif. Organisés avant ou après chaque séance officielle, comme le weekend, les caucus préparent les nouveaux à la connaissance de l'ONU et aux techniques d'intervention en séance. S'y rendent les autochtones et les ONG, mais pas les officiels des secrétariats onusiens, ni les États, sauf invitation expresse. Pour une description de ce mécanisme, voir Irène Bellier, «Partenariat et participation des peuples autochtones aux Nations unies: intérêt et limites d'une présence institutionnelle », dans Catherine Neveu, Démocratie participative, cultures et pratiques, Paris, L'Harmattan, 2007, p. $175-192$.

8. George E. Marcus, Ethnography through Thick and Thin, Princeton, Princeton University Press, 1998.
} 
les fonctionnaires internationaux et les représentants des États, ainsi que le style des relations de travail tranchent avec ce que l'on observe dans d'autres organisations internationales ${ }^{9}$. Ces pratiques sont emblématiques de l'utopie de la réconciliation que représente la Déclaration. De fait, la participation de ces acteurs à la négociation et la possibilité qui leur est offerte de s'exprimer au nom de leur organisation, de leur peuple et du collectif peuples autochtones sont une particularité saillante du dispositif onusien qui leur est consacré: «C'était la première fois que les Nations unies autorisaient la société civile (acteurs non étatiques) directement affectée par la décision politique à venir à la table négocier l'instrument lui-même ${ }^{10}$. Cette expérience ne se limite ni au temps de cette négociation ni à l'espace de son déroulement au sein des groupes de travail de la Commission des droits de l'homme ${ }^{11}$. Les conditions de possibilité d'une telle expérience subjective nous intéressent car les représentants autochtones tirent des modalités de leur reconnaissance internationale comme sujets de droits collectifs une légitimité qui leur permet de poursuivre des actions sur différents terrains.

Nous proposons ici de lier les formes de la saisine des Nations unies par des leaders autochtones aux modalités de construction institutionnelle de l'acteur politique agissant au nom des peuples autochtones, pour aborder in fine les implications de la reconnaissance comme « peuple » dans la gouvernance des « questions autochtones ».

\section{Bref aperçu ethnographique de la scène onusienne dédiée aux questions autochtones}

\section{Des rendez-vous annuels}

Genève, 22 juillet 2002, 8h du matin. Une file d'attente se forme à l'entrée de la Villa des Feuillantines où les délégués autochtones viennent récupérer leurs badges. Certains ont déjà rempli le formulaire qu'ils remettent en échange de ce sésame qui leur permettra de circuler dans le Palais des Nations, le temps de la session du GTPA. D'autres en sont encore à lire ledit formulaire bilingue (français/anglais), à sortir leur passeport, à renseigner les rubriques. Les participants des années précédentes aident les nouveaux, les hispanophones, les russophones et ceux pour qui les langues officielles de

\footnotetext{
9. I. Bellier, « Une approche anthropologique de la culture des institutions », dans Marc Abélès, Henri-Pierre Jeudy (dir.), Anthropologie du politique, Paris, Armand Colin, 1997, p. 129-161.

10. Nigel Crawhall, «Africa and the UN Declaration on the Rights of Indigenous Peoples », The International Fournal of Human Rights, 15 (1), 2011, p. 16 (nous traduisons).

11. Devenue en 2006 le Conseil des droits de l'homme.
} 
l'ONU ne sont qu'une langue secondaire. Certains ont un attaché-case bien rempli, d'autres une simple pochette et un crayon. Une jeune femme ogyek, appuyée au chambranle de la porte d'entrée de la villa, attend que la petite pièce d'environ $20 \mathrm{~m}^{2}$ se vide pour entrer à son tour. Embrassades, rires, le plaisir de se retrouver est manifeste. Les francophones sont peu visibles, bien que l'on soit à Genève. Par petits groupes, les participants se rendent au Palais Wilson, situé plus haut, en face du grand bâtiment de la Croix-Rouge. Aucune signalétique. Les nouveaux se contentent de suivre les anciens vers la salle où se tiennent les réunions du GTPA. Située en rez-de-jardin, celle-ci est voisine du Serpent, la cafétéria où les délégués se retrouvent pour discuter en marge des séances de travail.

New York, 16 mai 2011, 8h du matin. Une longue file d'attente s'étire sur le trottoir devant la grille d'entrée du siège des Nations unies et la série de drapeaux des États membres. Les personnes qui la composent illustrent la diversité culturelle de la planète par leurs vêtements et leurs coiffes qui tranchent avec l'ensemble cosmopolite des New-Yorkais, lesquels au demeurant les ignorent ${ }^{12} .8 \mathrm{~h} 30$. Un agent autoritaire, qui ne connait manifestement pas ce public, entrouvre la grille; la file se reforme à l'intérieur du sas de contrôle où, en ce jour d'ouverture de la dixième session de l'Instance permanente sur les questions autochtones (ci-après l'Instance), chacun se défait, comme dans les aéroports, de ses bijoux, plumes, perles, clochettes, couteaux cérémoniels, tambours, chapeaux, ceintures et pochettes. Les participants répéteront ces gestes quotidiennement durant deux semaines. Les jours de pluie, tout est plus compliqué. Les mallettes et sacs à dos sont remplis ; ordinateurs, caméras et appareils photo passent lentement sous le scanner. En dix ans, la panoplie du délégué autochtone s'est enrichie des outils de la communication moderne. Un peu plus loin, le groupe défile devant un comptoir où des fonctionnaires en civil des Nations unies délivrent un papier en échange de la lettre d'acceptation de la pré-inscription. Ce papier passe ensuite entre les mains d'agents en uniforme qui vérifient les données, prennent une photo de chaque délégué et fabriquent le badge qui permettra à celui-ci d'accéder aux salles dédiées aux questions autochtones mais pas à la salle du Conseil de sécurité. Des agents de sécurité surveillent les parcours.

12. Cette attitude contraste avec celle des Genevois qui ont «adopté » les délégués autochtones depuis qu'en 1923 Deskaheh, chef cayuga de la Fédération iroquoise des six nations, est venu à Genève pour faire reconnaître son peuple par la Société des Nations. Son action a été suivie en 1925 par celle de T.W. Ratana, leader spirituel maori de Aotearoa-Nouvelle-Zélande. Cf. Joëlle Rostkowski, « Deskaheh et la Société des Nations. Le Peau-Rouge demande justice », dans Musée d'ethnographie, Le visage multiplié du monde. Quatre siècles d'ethnographie à Genève, Genève, 1985, p. 151-167. 
Ces deux descriptions, notées in situ à dix ans d'écart, montrent que la présence des autochtones aux Nations unies est un événement toujours aussi important. Les délégués ne s'essoufflent pas, signe d'une détermination qui se forge dans l'expérience locale mais aussi indicateur que quelque chose se passe dans ce creuset du monde. Aux côtés des leaders et des organisations historiques qui se retrouvent depuis la fin des années 1970, de nouveaux venus intègrent chaque année le collectif. Ils font leurs des pratiques institutionnelles qu'ils apprennent des anciens et contribuent à faire évoluer. On note aussi que les fonctionnaires nationaux et internationaux se connaissent bien et rencontrent facilement les représentants autochtones. Le champ du dialogue institutionnalisé n'est pas seulement un lieu de circulation de paroles officielles. Occupé par des personnalités qui éclairent continument le présent et la construction du futur de leurs expériences du passé, il identifie cette scène des Nations unies comme un espace singulier dans l'ensemble des organisations internationales.

D'une année à l'autre, les mêmes gestes se reproduisent avec quelques changements. La présentation des rapports est plus visuelle grâce aux nouvelles technologies, l'enregistrement des participants s'est modernisé, les comptes rendus sont plus précis, la presse est mobilisée, les systèmes de sécurité ont été renforcés, à New York comme à Genève. Pour résoudre les difficultés posées par les techniques de contrôle à des personnes qui refusaient qu'on les touche ou qu'on leur interdise de porter leurs objets sacrés et qui ont pris la parole pour dénoncer « ces manifestations visibles d'une discrimination à leur encontre ${ }^{13}$, des négociations internes ont eu lieu, en 2007, qui permettent depuis à une Kichwa de l'Équateur de garder son chapeau pour la photo du badge, à un Mapuche du Chili de soustraire son tambour sacré du passage aux rayons $\mathrm{X}$, à des Papous d'introduire des lances dans l'enceinte, à une délégation nord-amérindienne de réaliser un rituel de fumigation...

Quelle importance faut-il accorder à ces pratiques? À première vue, elles peuvent paraître folkloriques, mais l'enquête montre qu'elles se combinent avec des prises de parole très politiques, des manières originales de faire consensus et des analyses techniques pointues. Elles représentent l'un des modes d'interaction entre groupes socialement constitués qu'évoque F. Barth dans son essai sur les frontières ethniques ${ }^{14}$. Elles nous permettent d'analyser cette scène internationale comme un lieu de renégociation des altérités, où la 
formation d'un «nous», peuples autochtones, rencontre l'expression d'un «nous », communauté internationale.

\section{Des statuts différents?}

Les délégués des organisations à statut consultatif ${ }^{15}$ arborent un badge à validité annuelle qui les dispense de la pré-inscription et facilite leur circulation, comme pour les fonctionnaires des Nations unies ou des États. Cette distinction encourage certaines organisations autochtones à se lancer dans les démarches, assez fastidieuses, de demande de ce statut auprès du Conseil économique et social (ECOSOC). La plupart d'entre elles se contentent cependant de la pré-inscription auprès du secrétariat des groupes de travail. L'administration onusienne a allégé son dispositif pour limiter les obstacles posés par certains États membres à la participation autochtone.

L'opération de comptage des participants est précise. On sait par exemple qu'à la neuvième session de l'Instance en 2010 étaient représentés 79 États membres, le Saint-Siège, 40 organes des Nations unies (agences spécialisées et organisations intergouvernementales), 17 organisations de peuples autochtones (OPA) à statut consultatif, $58 \mathrm{ONG}$ à statut consultatif, 188 organisations autochtones sans statut particulier et 32 institutions académiques, soit 415 entités composées chacune d'une délégation d'une ou de plusieurs personnes, et un total de 1500 personnes ${ }^{16}$. En revanche, le classement de ces entités change selon les organes concernés, et d'une année à l'autre. Ainsi, le Mécanisme expert sur les droits des peuples autochtones (MEDPA) distingue la catégorie «ONG, ainsi que organisations, peuples et nations autochtones» des catégories «universitaires et experts en questions autochtones», «institutions nationales de droits de l'homme», « organisations intergouvernementales, régionales et mécanismes droits de l'homme », « organes des Nations unies - agences spécialisées, fonds, programmes, mécanismes », «États membres » et «États non membres » (Saint-Siège). L'Instance, quant à elle, distingue désormais «États membres », «États non membres », « organes des Nations unies, agences spécialisées et organisations intergouvernementales », « organisations de peuples autochtones», « organisations de peuples autochtones à statut consultatif », « ONG à statut consultatif », « ONG sans statut consultatif », « organisations universitaires » et tout récemment « organes représentés par

15. Une quarantaine d'organisations autochtones et d'organisations dites de soutien bénéficient du statut consultatif soit général, soit spécialisé, soit sur liste (roster).

16. Notes de terrain, New York, 18 mai 2011. 
des parlementaires autochtones» ainsi que «institutions nationales des droits de l'homme ». Mais une organisation comme l'International Indian Treaty Council a été successivement classée comme «OPA à statut consultatif » (UNPFII 2003, 2006), « ONG à statut consultatif » (UNPFII 2009) ou « OPA» (UNPFII 2010). En 2011, le Comité des ONG recommandait à l'ECOSOC de lui reconnaître le statut le plus élevé, à savoir le statut consultatif général, jamais attribué jusque-là à une ONG autochtone. Au-delà de ces classifications, l'Instance entend mettre en valeur son rôle pivot dans la promotion du dialogue entre les représentants des États, ceux des organisations intergouvernementales et des agences spécialisées, et les organisations autochtones.

\section{Un espace de travail collectif}

Tous sont observateurs, sauf les membres experts des organes, qui, à l'aide des secrétariats, animent les sessions, produisent les documents d'étude et adoptent les rapports. Le temps étant précieux, tout le monde ne peut pas prendre la parole. Face à l'affluence, les interventions sont limitées parfois à deux minutes ; cela pousse à l'expression collective, le regroupement d'organisations offrant un temps de parole supérieur, soit cinq minutes. Les États n'interviennent que s'ils ont vraiment un point à développer. La différence entre les États et les autochtones est que les premiers n'ont pas toujours besoin de s'inscrire pour exercer leur droit à la parole. Par ailleurs, depuis les premières rencontres des années 1980, il est d'usage que les ONG de soutien laissent la parole aux représentants autochtones.

Si l'on distingue dans la salle ceux qui ont une expérience de ces forums de ceux qui sont trop impressionnés pour parler, rien ne permet de remarquer les différentes affiliations statutaires (OPA ou ONG, à statut consultatif, général ou non). Certes, les représentants des États, des organisations intergouvernementales et des agences spécialisées s'assoient aux places qui portent leur nom. Une géographie des lieux peut donc être établie : à la tribune, le/la président/e du Groupe de travail ou de l'Instance, les conférenciers invités, le secrétariat; au premier rang de l'hémicycle, les autres membres des organes considérés ; derrière eux, les délégués des États, des agences et des organisations intergouvernementales, puis les représentants des organisations autochtones et tout au fond les ONG de soutien et les centres universitaires ou de recherche. On observe toutefois que les autochtones s'assoient souvent aux places réservées à leurs États respectifs lorsque le représentant officiel est absent. Les délégations de certains États comprennent aussi des autochtones, lesquels composent parfois la délégation dans son intégralité, comme pour la 
Bolivie depuis 2009. Ces pratiques brouillent l'opposition (affichée durant la négociation de la Déclaration) entre les États qui, disposant du pouvoir de voter, sont les sujets reconnus du droit international, et les délégations autochtones qui, en formulant leurs préoccupations, recherchent cette reconnaissance internationale.

Dans la salle règne un joyeux brouhaha, malgré les rappels à l'ordre du président de séance. Les délégués entrent et sortent, participent aux débats ou profitent de ces rares moments de rassemblement pour finaliser le texte d'une intervention collective, obtenir les signatures nécessaires à la validation de leur document, rencontrer les représentants de leur État, se promener aussi... Ils et elles se prennent en photo, filment leurs interventions, distribuent des invitations pour les événements parallèles qui se tiendront entre les séances plénières. Une montagne de données circule en permanence sous forme de documents de travail de l'organe considéré ${ }^{17}$, d'affiches, de livres, de coupures de presse, de livrets d'organisations, de CD et de DVD. La collecte de ces données, dont les stocks s'épuisent rapidement, est une activité continue de tous les participants.

\section{La construction d'un acteur collectif par la production d'une identité générique}

L'ONU dénombre 370 à 400 millions d'autochtones dans près de 90 États (UNPFII 2010). Les indicateurs démographiques sont stables bien que l'Organisation n'ait pas les moyens de commander un recensement global et que le nombre d'États concernés soit passé de 70 à 90 entre 2002 et 2009. Compte tenu de la diversité des manières de nommer et de compter les populations concernées, une sorte de consensus s'est formé autour des critères avancés par José Martínez Cobo dans son Étude de la question de la discrimination à l'égard des populations autocbtones ${ }^{18}$. Ces dernières sont « des peuples et des nations qui présentent une continuité historique avec les sociétés précédant la conquête et la colonisation de leurs territoires, qui se considèrent comme distincts des autres secteurs de la société dominant ces territoires totalement ou partiellement (...) [et] sont déterminés à préserver, développer et transmettre aux générations futures leurs territoires ancestraux et leur identité ethnique, sur la base de leur existence continue en tant que peuple,

17. Un corpus documentaire rédigé dans les six langues de travail de l'ONU (anglais, arabe, chinois, espagnol, français, russe) est distribué aux participants. Il contient les études et rapports examinés en séance, en rapport avec les thématiques mises à l'agenda l'année précédente.

18. Auteur avec Augusto Willemsen Diaz de la première étude commanditée par l'ECOSOC en 1972, finalisée et publiée en 1986. José Martínez Cobo, Study of the Problem of Discrimination against Indigenous Populations, E/CN.4/ sub 2/1986/87 add 1-4, ONU, 1986. 
en accord avec leurs propres systèmes culturels, leurs systèmes juridiques et leurs institutions sociales ». Si chacun des termes de cette définition est sujet à interprétation, l'approche multicritère offre l'intérêt de dés-essentialiser les sujets concernés. Le processus contribue à créer une identité générique peuples autochtones que nous considérons principalement comme catégorie politique et relationnelle.

Cette identité globale heurte les manières de s'identifier propres à chacun de ces peuples, qui sont évidemment variées, ainsi que les dispositifs constitutionnels, juridiques et politiques des États qui autorisent ou pas la reconnaissance de ces entités. Avec des terminologies variant selon leur histoire politique, la plupart des États les connaissent comme «tribus», «minorités nationales ou linguistiques », «groupes ethniques » ou par des appellations péjoratives (« secteurs arriérés »). Mais plusieurs États d'Amérique latine les reconnaissent comme «nations», «peuples» ou «nationalités» ${ }^{19}$. Du lexique élaboré pour construire un consensus international, le GTPA a retenu l'expression peuples autochtones pour inclure les centaines de peuples vivant dans les États indépendants et colonisés. Cette traduction de indigenous peoples/pueblos indigenas a été choisie pour contourner l'inadéquation du mot «indigène » hérité du statut personnel concédé aux populations locales par la France coloniale. Par-delà la critique que suscite le terme « autochtone » en raison de « conflits de subjectivation » notamment en Afrique ${ }^{20}$, le terme «peuple» plutôt que «population »a constitué le véritable enjeu, au moment où le GTPA se saisissait des dossiers dans les domaines pour lesquels il devait élaborer des normes. Le substantif « peuple » est bien plus important, dans l'échelle des valeurs onusiennes, que le qualificatif «autochtone». À l'issue de débats animés, il a été décidé qu'aucune liste de peuples n'accompagnerait la Déclaration, les représentants autochtones martelant le principe de leurs responsabilités en matière d'identification. Cependant, les organisations internationales comme les États poursuivent un travail de clarification. Ainsi, le Groupe de travail de la Commission africaine des droits de l'homme et des peuples précise les entités

\footnotetext{
19. En Amérique latine, le « réveil indien » se traduit par une réappropriation des noms propres, par des changements graphiques et par l'entrée dans les constitutions nationales de références aux peuples indigènes en tant que « nation » et « nationalité » (Équateur) ou (comme en Bolivie) naciones y pueblos indigena originario campesinos, formule composite qui ne cesse de poser problème. Voir Marco Aparicio (ed.), Los derechos de los pueblos indígenas a los recursos naturales y al territorio. Conflictos y desafíos en América latina, Barcelone, Icaria, 2011. Cette dynamique s'observe également en Afrique autour de la réfutation du terme « Pygmées », actée par une loi de la République du Congo adoptée fin 2010.

20. Jean-François Bayart, Peter Geschiere, Francis Nyamnjoh, «Autochtonie, démocratie et citoyenneté en Afrique », Critique internationale, 10, janvier 2001, p. 177-194.
} 
relevant de l'approche onusienne - chasseurs-cueilleurs, pasteurs nomades, habitants des forêts ${ }^{21}$ - et nomme leurs sociétés.

Sur la scène onusienne, les délégués autochtones réaffirment le nom de leur peuple, ouvrent leurs discours par une adresse dans leur langue, dénoncent la discrimination, expliquent les processus de récupération identitaires et linguistiques $^{22}$ et procèdent aujourd'hui à la traduction de la Déclaration dans les langues non officielles.

Face à ces différentes pratiques, on constate des décalages entre la catégorie internationale qui établit une communauté de statut juridique (« les peuples autochtones ont le droit de...»); les catégories étatiques qui définissent à leurs manières les sujets des politiques publiques ; les catégories des anthropologues qui connaissent des ethnonymes, des cultures ou des langues que le droit international n'influence qu'à la marge ; et les catégories que les délégations autochtones utilisent en naviguant entre singulier et collectif, comme en témoignent ces deux interventions parmi tant d'autres : « Mon nom est X, j'appartiens au peuple Kaweshkar, en voie d'extinction, localisé dans la région $12 \mathrm{du}$ Chili (...) et nous devons inclure le peuple-frère Yagan qui compte actuellement une seule survivante ${ }^{23}$; «Je représente le peuple Oraon, de Jharkand, Inde. Madame la présidente, je voudrais vous présenter l'histoire du développement de mon peuple dans le contexte de la discussion sur la protection et la promotion des droits des peuples indigènes et tribaux de ce distingué groupe de travail $\gg^{24}$.

\section{La formation du « Nous, peuples autochtones"}

La collection de témoignages, l'instruction des problèmes et l'adoption de la Déclaration conduisent à la formulation «nous, peuples autochtones » qui fait écho aux premiers mots de la Charte des Nations unies. Ce « nous » est représenté sur le site web de l'Instance par deux logos superposés. Le premier suggère la rencontre entre les peuples du monde sous la forme d'une poignée de

\footnotetext{
21. CADHP/ACHPR, Rapport du Groupe de travail d'experts sur les populations/communautés autochtones, bilingue, Copenhague, IWGIA, 2005.

22. Les représentants autochtones articulent la défense de leurs droits avec le dispositif de la Convention 169, principalement en Amérique latine où quinze pays l'ont ratifiée, contre seulement quatre pays en Europe, un en Afrique, un en Asie, et un en Océanie. Ils s'inscrivent dans la promotion de la diversité culturelle que l'UNESCO poursuit depuis la Déclaration sur la diversité culturelle (2001) et les Conventions sur la sauvegarde du patrimoine culturel immatériel (2003) et sur la diversité des expressions culturelles (2005).

23. UNPFII, 2004 (nous traduisons).

24. GTPA 2001. Suit l'énumération en une centaine de mots des problèmes de déplacement dus à des projets de développement et à la perte de moyens de subsistance, pour demander le droit à l'autodétermination et un empowerment, afin que les peuples autochtones puissent décider de leurs affaires.
} 
main de couleurs différentes, au centre d'une évocation de la terre, de la mer, des montagnes, de l'air, éclairée par un soleil et entourée d'une couronne de feuillage verte; le second inscrit «We, the Peoples» au centre du logo des Nations unies en filigrane. Avec la référence aux éléments naturels, ce «nous » est une prise de position publique, comme le manifestèrent lors de la conférence de Vienne sur les Droits humains (1993) les délégués qui brandirent un $\ll S \gg$ pour affirmer la nature collective de l'appartenance à un peuple ${ }^{25}$.

\section{Subjectivation et visibilité}

Le monde autochtone onusien est diversifié en termes d'origine, d'âge, de socialisation des individus, ce qui rend d'autant plus signifiantes les convergences observées dans les pratiques organisationnelles et discursives. La représentativité des délégués est rarement questionnée, mais il arrive que des États accusent certaines organisations d'être instrumentalisées par «l'extérieur ${ }^{26}$. Cette altérité-là se renégocie via un dispositif qui vise l'inclusion des communautés en question dans un jeu démocratique.

Les délégués viennent des capitales, où ils ont acquis une formation universitaire et militante, comme de lieux très reculés, tels un instituteur shipibo du Pérou, chargé de recueillir les moyens d'une éducation bilingue; une déléguée ma'ohi, qui met en cause les traités avec la France ; un représentant san embarqué par la délégation du Botswana pour une opération de communication politique... Les premiers attestent une capacité à forger des discours très politiques en dominant le langage juridique. Les seconds, parmi des centaines d'autres dont le déplacement est facilité par les Nations unies ${ }^{27}$, témoignent des difficultés à comprendre les formes institutionnalisées du débat. Ils apprennent les codes peu à peu, guidés par les anciens dont certains sont présents depuis $1977^{28}$. Si les délégués autochtones perçoivent la scène internationale comme le moyen de faire connaître leur cause pour influencer les autorités du pays, chacun raconte une histoire personnelle sur ses raisons d'être là, et les récits convergent vers quelques motifs : « Cela fait des années que l'on demande la mise en œuvre des accords de paix» (délégué des Chittagong Hill Tracks - Bengladesh, auquel fait écho un délégué maya du

25. Erica-Irene A. Daes, Indigenous Peoples. Keepers of our Past-Custodians of our Future, Copenhague, IWGIA, 2008, p. 21.

26. Selon les règles des Nations unies, tout État peut s'opposer à l'expression de personnes ou d'organisations lorsque sa politique est mise en cause. Dans les situations que nous avons observées, le Bengladesh, la Birmanie, la Chine, l'Indonésie, la Thaïlande et le Vietnam ont exprimé une critique ponctuelle.

27. Fonds de contributions volontaires des Nations unies pour les populations autochtones, abondé par les États et cogéré par les autochtones.

28. Date de la première réunion des ONG à Genève sur « la discrimination dans les Amériques ». 
Guatemala); «J'ai des observations à faire à la Banque mondiale » (délégués papou et baka du Congo, plusieurs autres) ; « On m'a chargée de présenter la plainte de notre peuple contre le projet de barrage à Belomonte » (déléguée du Xingu, Brésil) ; «Je viens rencontrer le rapporteur spécial » (délégation kanak de Nouvelle-Calédonie); « Le tourisme/la mine/la route/le barrage détruit nos sites sacrés $\gg$ (plusieurs délégués) ${ }^{29} \ldots$

L'expérience de l'ONU commence dans les couloirs, se prolonge en séance plénière et se fortifie dans les circuits de formations des leaders. Elle confère au délégué une légitimité et des moyens d'action mobilisables localement. Le décalage entre scènes internationales et nationales, souligné par les acteurs comme par les anthropologues, n'entame pas le pouvoir d'attraction des Nations unies qui configurent le lieu où un sujet collectif, «nous, peuples autochtones $»$, s'énonce.

Ce «nous » s'appuie sur une histoire et plutôt des histoires : celle des pionniers dont la geste est popularisée sur le site de l'Instance; celle des autochtones qui restituent dans les conversations privées ou à la tribune l'émotion des premières rencontres; celle qu'écrivent aujourd'hui les « embrayeurs de changement », comme Erica-Irene A. Daes, présidente du GTPA durant dixhuit ans ${ }^{30}$, les négociateurs de la Déclaration ${ }^{31}$ et/ou les observateurs du processus depuis les années $1970^{32}$. Ces récits complètent les travaux des anthropologues et des historiens ${ }^{33}$ sur le mouvement international et l'élargissement des problématiques politiques de l'autochtonie.

Il s'appuie également sur des catégories linguistiques. Par la tradition orale et grâce aux nouvelles technologies, une filiation métaphorique est construite entre un «père fondateur ${ }^{34}$, dont l'action est poursuivie par des chefs

\footnotetext{
29. Synthèse d'entretiens.

30. E.-I. A. Daes, auteur de l'étude consacrée à la Souveraineté permanente des peuples autochtones sur les ressources naturelles (E/CN.4/Sub.2/2004/30, 13 juillet 2004, http://www.un.org/esa/socdev/unpfii/documents/Report $\% 20$ by $\% 20$ Erica $\% 20$ Irene $\% 20$ A. $\% 20$ Daes $\% 20 \% 28$ French $\% 29$. pdf), relate cette histoire dans E.-I. A. Daes, Indigenous Peoples. Keepers of our Past-Custodians of our Future, op. cit..

31. C. Charters, R. Stavenhagen (eds), Making the Declaration Work: The United Nations Declaration on the Rights of Indigenous Peoples, op. cit..

32. Jens Dahl, IWGLA. A History, Copenhague, IWGIA, 2010.

33. Entre autres, Ronald Niezen, The Origins of Indigenism: Human Rights and the Politics of Identity, Los Angeles, The University of California Press, 2003 ; Henry Minde (ed.), Indigenous Peoples: Self-determination, Knowledge and Indigeneity, Delft, Eburon Publishers, 2008 ; Quentin Gausset, Justin Kenrick, Robert Gibb, « The Uses and Misuses of "Indigeneity" and "Autochthony" », Social Antbropology, 19 (2), 2011, numéro spécial.

34. Deskaheh est évoqué par des autochtones du monde entier qui ne savent pas ce qu'est la Fédération iroquoise des six nations (Haudenesaunee). Lors de leur première prise de parole, ils félicitent la présidence de l'organe et rendent hommage aux Haudenesaunee (terme iroquois signifiant « les habitants des longues maisons »), héritiers spirituels du territoire tuscarora sur lequel s'est développé l’État de New York. Également célébré dans les discours des secrétaires généraux de l'ONU, depuis Kofi Annan qui, en 2006, rendit hommage à la détermination des peuples autochtones, Deskaheh occupe une place privilégiée dans un imaginaire partagé par l'institution et les militants.
} 
nord-amérindiens, et des «fils et filles en autochtonie»du monde entier. Cette construction établit les principes d'une communauté horizontale entre des activistes qui se reconnaissent « frères » et « sœurs », en termes d'adresse et de référence. Les propos des autochtones dessinent ainsi un vocabulaire de la parenté (fictive) qui distingue les ancêtres, propres à chacun; les esprits tutélaires partagés que sont la « terre mère » et le « père créateur » invoqués lors des cérémonies d'ouverture et de clôture ; enfin, cette parenté collatérale qui s'enracine dans une expérience commune ${ }^{35}$. Au-delà des distinctions de langue, d'âge ou d'origine, le discours de la fraternité établit les principes d'une solidarité qui s'étend aux personnes offrant leur soutien.

\section{Quête d'égalité}

Le «Nous, peuples autochtones», sans État ni patrie, configure un ensemble ouvert à « l'autre ${ }^{36}$ afin d'être reconnu dans l'ensemble de l'humanité. La cohérence est remarquable dans les pratiques dialogiques, discursives et cérémonielles qui manifestent le désir d'inclusion. En termes cérémoniels, deux éléments marquent la spécificité autochtone : la pratique d'oraisons à l'ouverture et à la clôture des sessions de travail, réalisées le plus souvent par des leaders spirituels nord-amérindiens, mais pas exclusivement ; la réalisation de performances combinant les « cultures traditionnelles » de peuples représentant les régions dites « socioculturelles » structurant le monde autochtone onusien ${ }^{37}$. Ces événements intègrent les non-autochtones et rendent visibles l'enjeu de la protection de la diversité culturelle.

En termes de dialogue, l'association aux processus de décision s'affirme sur la scène internationale par la mise en place de l'Instance dont les membres sont choisis à parité par les représentants des groupes d'États ${ }^{38}$ et par les organisations autochtones des régions précitées; dans les espaces politiques locaux, à partir de l'approche instruite par l'étude du MEDPA sur «le droit des peuples autochtones à participer aux processus de décision $»^{39}$, et enfin à travers

35. Notes de terrain.

36. Sous l'angle d'une altérité indigène incluse dans un « nous national ». Voir, à propos du Mexique, Paula López Caballero, « Altérités intimes, altérités éloignées : la greffe du multiculturalisme en Amérique latine », Critique internationale, 51, 2011, p. 129-149.

37. Afrique, Amérique-Centre-Sud-et-Caraïbes, Amérique du Nord, Arctique, Asie, Europe centrale et orientaleFédération de Russie-Asie Centrale-Transcaucasie, et Océanie.

38. I. Bellier, «The Declaration of the Rights of Indigenous Peoples and the World Indigenous Movement», Griffith Law Review, 14 (2), 2005, p. 227-246 ; I. Bellier, « Le projet de Déclaration des droits des peuples autochtones et les États américains : avancées et clivages », dans C. Gros, M.-C. Strigler (dir.), Être indien dans les Amériques - Spoliations et résistance. Mobilisations etbniques et politiques du multiculturalisme, op. cit., p. 27-41.

39. A/IIRC/EMRIP/201i/2. 
l'examen du « droit au consentement libre, préalable et informé » sur le respect duquel insistent les organisations autochtones comme une forme d'exercice du droit à l'autodétermination. Le débat sur ce thème traverse les Nations unies jusqu'à la Banque mondiale qui cependant donne au concept une sérieuse inflexion en remplaçant le terme «consentement» par celui de « consultation ». La volonté d'inclusion des autochtones à des fins de dialogue s'appuie aussi sur la constitution de délégations représentant la diversité du monde autochtone et stimule la construction de Parlements et de réseaux transnationaux. Ces derniers facilitent l'échange d'expériences ${ }^{40}$ et augmentent la crédibilité des organisations dans les négociations des forums spécialisés sur l'eau, l'avenir des peuples des forêts tropicales ou le changement climatique... En ce qui concerne les discours, on observe une normalisation des prises de parole depuis que, dans les années 1980, la présidente du GTPA a rappelé à l'ordre les autochtones et les représentants des États pour que les autochtones évitent de choquer les représentants des États et que ceux-ci les respectent. Une telle disposition était indispensable pour élaborer le triple consensus nécessaire à l'adoption de la Déclaration: entre États, entre organisations autochtones, entre États et organisations autochtones. Nous ne pouvons analyser ici les discours autochtones dans leurs contextes d'énonciation, mais ils convergent sur la dénonciation de situations devenues communes ${ }^{41}$. À partir des cas présentés par les observateurs, les experts instruisent les situations dans un langage juridique, nourri par la connaissance du droit international et des décisions de justice. C'est ainsi qu'aujourd'hui la conception de politiques et d'indicateurs «culturellement adaptés », coproduits par des experts autochtones et des agences onusiennes pour répondre à l'évaluation des places sur l'échelle du développement, conduit à la formule du « développement avec culture et identité », reprise par les banques de développement ${ }^{42}$.

Malgré la variété des déclarations de délégués s'exprimant avec plus ou moins de facilité dans les langues officielles, une voix collective se dégage, telle une musique dominée par la rhétorique des droits de l'homme et ponctuée d'analyses très techniques. Cela distingue le site dédié aux questions autochtones de tous les sites institutionnels que nous avons pu observer.

\footnotetext{
40. Plusieurs organisations autochtones se situent au niveau régional : en Amazonie, la COICA regroupe 9 organisations unitaires ou fédérales sur 9 pays ; en Afrique, l'IPACC rassemble 150 organisations sur 22 pays ; en Asie, l'AIPP regroupe 39 organisations dont 9 fédérations nationales sur 14 pays.

41. Les déplacements forcés liés à la réalisation de grands ouvrages dits de développement, les spoliations territoriales pour le compte d'entreprises minières ou agro-industrielles, la pollution des eaux et la dégradation de la santé, la pauvreté urbaine, l'exclusion sociale et la marginalisation socioéconomique.

42. Victoria Tauli-Corpuz, Leah Enkiwe-Abayao, Raymond de Chavez (eds), Towards an Alternative Development Paradigm: Indigenous People's Self-determined Development, Baguio, Tebtebba Foundation, 2010.
} 


\section{La reconnaissance des sujets collectifs dans la gouvernance des questions autochtones}

Depuis les années 1970, on est passé d'une situation où les «autres» (les Occidentaux) parlaient des « populations indigènes » à ce moment de prise en charge de soi par lequel ces entités construisent, par la voie des leaders qu'elles se reconnaissent, une capacité à parler elles-mêmes des peuples autochtones.

Quelles que soient les critiques que l'on puisse émettre quant à la représentativité des sujets, la catégorie « expert » donne aux représentants autochtones le moyen de produire des études qui ont un effet dans le champ institutionnel, comme en témoignent les changements que les États opèrent, par exemple en matière d'éducation de la jeunesse autochtone ${ }^{43}$. En témoigne aussi un nouveau style de publications dans lequel, en plus du statut d'acteurs/témoins, certains auteurs sont marqués d'une double affiliation, dans les organisations autochtones et l'université ou dans l'expertise internationale et le champ de l'autochtonie. Ce processus montre comment le sujet singulier (l'auteur) s'articule au sujet collectif (les peuples autochtones).

Certes, la construction de l'autochtone comme sujet ne peut être attribuée de façon exclusive à la machine onusienne, mais l'Organisation constitue la plateforme à partir de laquelle les peuples autochtones s'énoncent comme sujets collectifs par une approche générique de leurs problèmes. Elle représente le point d'articulation de dispositifs éclatés entre des agences spécialisées dont les programmes d'action génèrent toutes sortes d'expectatives et de frustrations.

\section{De l'espace de la reconnaissance...}

La représentation des autochtones, à partir des années 1980, dans les organes comme le GTPA, le GTPD, l'UNPFII et le MEDPA, ont permis que se constitue le «nous » évoqué plus haut. Mais elle ne se retrouve pas dans les organes de décision tels que le Conseil économique et social, l'Assemblée générale, le Conseil de sécurité et le Conseil des droits de l'homme. De même, dans les organes de traités, les Organisations (l'UNESCO, l'OIT) ou dans les Fonds, la participation des interlocuteurs autochtones est sélective et marginale.

\footnotetext{
43. L'Australie et le Canada ont chacun présenté des excuses nationales aux « Aborigènes et Premières Nations » pour les torts causés par la politique des pensionnats. Plus largement, sur la question des jeunes, voir Natacha Gagné, Laurent Jérôme (dir.), Feunesses autochtones. Affirmation, innovation et résistance dans les mondes contemporains, Rennes, Presses universitaires de Rennes, 2009.
} 
Cependant, la circulation des délégués autochtones dans ces institutions nourrit une demande de participation dans tous les lieux qui ne les consultent pas systématiquement pour la conception des programmes ou leur mise en œuvre. Sont principalement visées (et dénoncées) les Banques régionales de développement et les entreprises multinationales, extractives ou agroindustrielles, dont les projets sont à l'origine de la plupart des situations conflictuelles aujourd'hui.

La constitution de soi comme «peuple» doté d'une personnalité juridique en droit international peut déclencher un changement dans les relations avec l'État concerné, mais la Déclaration n'est pas directement applicable, même si elle contient une disposition engageant l'ensemble du système des Nations unies et les États membres à la rendre effective (art. 42). Le terme anglais « to redress », dont les traductions française (réparer) et espagnole (resarcir/compenser) ont été longuement débattues au GTPD en 2006, rend compte de l'enjeu à long terme du dispositif: il évoque une possible échappée des figures de la soumission qui habitent le portrait de l'indigène dans la pensée dominante.

\section{... à la gestion de la différence}

Un autre point d'intérêt se dégage de la scène observée quant à la possibilité de lier une approche globale par le droit et des programmes nationaux et catégoriels. Le Haut-Commissariat aux droits de l'homme (HCDH) s'efforce d'intégrer les questions autochtones dans les agences onusiennes. Cette politique se répercute dans les organisations intergouvernementales (l'Organisation des États américains ou l'Union européenne, entre autres). L'institution de l'Instance, dont le mandat est de formuler des recommandations aux États, répond à cet objectif dit de mainstreaming. Elle vise à transformer les discours en actes politiques, juridiques et techniques, afin de répondre à des situations de tensions et de conflits. Mais de quelle façon ?

L'ONU distingue dans l'ensemble des peuples autochtones des interlocuteurs légitimes qui construisent leur expertise dans le triple champ de l'appartenance à un peuple, de la connaissance technique et de la maitrise du langage et des rapports de pouvoir internationaux. Localement, les autochtones sont de plus en plus formatés pour répondre aux appels d'offre pour la réalisation de micro-projets. Leur incorporation sous contrôle contribue à scinder le sujet collectif peuples autochtones, politiquement délicat à gérer, pour appréhender techniquement les problèmes. Ainsi, chaque agence spécialisée se préoccupe qui du «travailleur indigène»(OIT), qui des «enfants autochtones » (UNICEF), des «femmes autochtones » (UNIFEM), de la 
«santé autochtone» (OMS) ou des «autochtones urbains» $50 \%$ de la population estimée selon UN-Habitat). Ces thèmes se substituent aux catégories d'opposition qui imprègnent le discours revendicatif («victimes/ colonisateurs », «dominés/dominants », « pauvres/riches »), mais dont la pertinence diminue à mesure que les questions autochtones s'exportent hors du domaine couvert par le HCDH. Ainsi, l'association des autochtones aux travaux du Comité intergouvernemental sur « la propriété intellectuelle relative aux ressources génétiques, aux savoirs traditionnels et au folklore», créé en 2000 par l'Organisation mondiale de la propriété intellectuelle (OMPI), génère une opposition inédite entre « détenteurs de savoirs traditionnels » et « détenteurs de brevets», à partir d'un jeu de mots sur «IPR » : Indigenous Peoples Rights/Intellectual Property Rights.

$\boldsymbol{L}_{\mathrm{a}}$ Déclaration des droits des peuples autochtones a conduit à la formulation rhétorique puissante de l'identité globale peuples autochtones, en tant que sujets de droits. Elle configure un nouvel imaginaire, en postulant une égalité de traitement entre ces derniers et les peuples (à État) des Nations unies, via la reconnaissance de droits collectifs dans le respect du droit international (art. 46).

L'émergence internationale des peuples autochtones a partie liée avec les formes de la globalisation et les techniques du néolibéralisme. Saisies comme contextes, ces dernières éclairent le déplacement des problématiques locales sur la scène globale, informent des transformations dans le champ des mobilisations et permettent de problématiser la fabrique de normes internationales. Les peuples autochtones ne sont pas une invention internationale mais le symbole d'une requalification opérant dans une série de tensions entre les espaces localisés des communautés et les espaces globalisés où interviennent leurs représentants. Entre le village local et le village global s'est constituée une communauté épistémique dont les acteurs se répartissent dans les espaces de décision. L'entrée des autochtones à l'ONU a scellé le début d'un processus d'institutionnalisation fondamental pour la construction d'une voix collective. Les représentants ont organisé cet espace de parole en se posant en acteur doué d'autonomie réflexive pour construire les moyens de la reconnaissance comme «peuple » en droit international.

Les Nations unies sont la scène du retour d'un sujet refoulé ${ }^{44}$. Le point de vue de ceux que l'on pensait assimilés par le développement d'un corps

44. Comme en témoignent les titres de deux publications : « The Return of the Native » de Adam Kuper, Current Anthropology, 44 (3), 2003, p. 389-401, et El regreso de lo indígena. Retos, problemas y perspectivas de Valérie Robin Azevedo, Carmen Salazar-Soler (ed.), Lima, Cusco, IFEA, CBC, 2009. 
stato-national conçu comme homogène ne peut plus être écarté par des effets de domination sociale et politique. Leur « différence » est une constituante de leur modernité ${ }^{45}$. Cependant, les institutions onusiennes forgent des interlocuteurs autochtones dans une configuration qui repose sur un processus de reconnaissance et de mutuelle dépendance : elles ont besoin d'eux en tant qu'experts pour légitimer leurs programmes comme ils ont besoin d'elles pour peser sur les conditions politiques locales.

La politique de reconnaissance poursuivie par le mouvement international a poussé, avec bien d'autres facteurs, les pays latino-américains à des changements constitutionnels significatifs du glissement vers le multiculturalisme puis vers la construction d'un État plurinational ${ }^{46}$. Des évolutions sensibles ont également lieu sur d'autres continents : aux Philippines, avec l'adoption d'une loi sur les droits autochtones (1997), et en République Centrafricaine, premier et unique pays d'Afrique à adopter la Convention 169 en 2010, comme le Népal avait été en 2007 le premier et unique pays d'Asie à la ratifier. Les effets de ces dispositifs sont lents, complexes à observer du point de vue ethnographique, et l'on note d'évidents décalages entre les positions des États sur la scène internationale et leurs pratiques politiques « domestiques ». L'enjeu pour les représentants autochtones reste de sortir du cercle des experts onusiens pour se positionner comme interlocuteurs légitimes des autorités nationales. L'enjeu pour le système onusien est de poursuivre dans le champ labellisé «droits des peuples autochtones » les réformes des systèmes nationaux ${ }^{47}$.

Irène Bellier est anthropologue, directrice de recherches au CNRS et directrice du Laboratoire d'anthropologie des institutions et des organisations sociales (LAIOS, IIAC, CNRS-EHESS). Depuis sa thèse sur les rapports entre les femmes et les hommes mai huna en Amazonie péruvienne (publiée en 1991), elle travaille à une anthropologie des institutions et du politique. De la formation des élites françaises aux enjeux de la diversité culturelle, ses recherches sur les institutions du pouvoir, le multiculturalisme et les politiques de développement l'ont conduite vers l'étude des transformations politiques et juridiques du statut des populations autochtones. Prenant pour terrain les Nations unies, elle étudie le mouvement international des peuples autochtones dans une perspective comparative et multiscalaire. Elle est actuellement Principal Investigator d'un projet subventionné par le Conseil européen de la recherche (Scales of Governance. The UN, the States and

45. C. Gros, «Ser diferente por (para) ser moderno, o las paradojas de la identidad. Algunas reflexiones sobre la construcción de una nueva frontera étnica en América latina », Análisis Político, 36, 1999, p. 3-20.

46. Raquel Z. Yrigoyen Fajardo (coord.), Pueblos indígenas : constituciones y reformas políticas en América latina, Lima, ILSA, IIDS, INESC, 2010.

47. Cet article s'appuie sur une recherche financée en partie par le Conseil européen de la recherche, dans le cadre du 7 e programme cadre (FP7/2007-2013 Grant Agreement ERC n² 249236) (www.sogip.ehess.fr). 
Indigenous Peoples: Self-determination at the Time of Globalization). Elle a publié, entre autres, Savoirs et politiques de développement. Questions en débat à l'aube du XXIe siècle (avec Vincent Geronimi, Jean-Jacques Gabas, Michel Vernières, Yves Viltard (dir.), Paris, Karthala, 2008). Voir l'ensemble de ses publications sur http://www.iiac.cnrs.fr/laios/ spip.php?article250. Adresse électronique : ibellier@ehess.fr 